\title{
Demonstration of Safe Termination of Megaampere Relativistic Electron Beams in Tokamaks
}

\author{
Cédric Reux $\oplus^{*}$ \\ CEA-IRFM, F-13108 Saint-Paul-les-Durance, France \\ Carlos Paz-Soldan 1 \\ General Atomics, P.O. Box 85608 San Diego, California 92186-5608, USA \\ and Department of Applied Physics and Applied Mathematics, Columbia University, New York, New York 10027, USA \\ Pavel Aleynikov \\ Max-Planck-Institut für Plasmaphysik, Greifswald, Germany \\ Vinodh Bandaruำ \\ Max-Planck-Institut für Plasmaphysik, Garching, Germany \\ Ondrej Ficker@ \\ Institute of Plasma Physics of the CAS, Za Slovankou 1782/3, 18200 Praha 8, Czech Republic \\ Scott Silburn \\ CCFE, Culham Science Centre, Abingdon, Oxon OX14 3DB, United Kingdom \\ Matthias Hoelzlø \\ Max-Planck-Institut für Plasmaphysik, Garching, Germany \\ Stefan Jachmich \\ ITER Organization, Route de Vinon sur Verdon, 13115 St. Paul Lez Durance, France \\ Nicholas Eidietis $(1)$ \\ General Atomics, P.O. Box 85608 San Diego, California 92186-5608, USA \\ Michael Lehnen (1) \\ ITER Organization, Route de Vinon sur Verdon, 13115 St. Paul Lez Durance, France \\ Sundaresan Sridhar@ \\ CEA-IRFM, F-13108 Saint-Paul-les-Durance, France \\ JET contributors ${ }^{\dagger}$
}

(Received 19 September 2020; revised 19 February 2021; accepted 16 March 2021; published 30 April 2021)

For the first time it is experimentally demonstrated on the JET tokamak that a combination of a low impurity concentration bulk plasma and large magnetohydrodynamic instabilities is able to suppress relativistic electron beams without measurable heat loads onto the plasma facing components. Magnetohydrodynamic simulations of the instability and modeling of the postinstability plasma confirm the prompt loss of runaways and the absence of regeneration during the final current collapse. These surprising findings motivate a new approach to dissipate runaway electrons generated during tokamak plasma disruptions.

DOI: 10.1103/PhysRevLett.126.175001

Published by the American Physical Society under the terms of the Creative Commons Attribution 4.0 International license. Further distribution of this work must maintain attribution to the author(s) and the published article's title, journal citation, and DOI.
Introduction.-Runaway electrons (REs) are created in thunderstorm clouds [1,2], solar flares [3,4], as well as during disruptions of tokamak plasmas [5]. The latter two situations share a close link to magnetic reconnection events [6], an ability to convert magnetic energy into 
kinetic energy and similar ranges of densities and electric fields [7-9]. In tokamak disruptions, REs reach energies up to $10 \mathrm{~s}$ of $\mathrm{MeV}$ in multi-mega-ampere beams [10-12] and lead to significant localized damage on plasma facing components (PFC) upon termination $[13,14]$. The kinetic and magnetic energy stored by a RE beam in future tokamaks will reach 10 s of megajoules $[15,16]$. A mitigation strategy is therefore mandatory [17].

Shattered pellet injection (SPI) [18] is presently the baseline RE mitigation actuator planned for the ITER tokamak. Its goal is to avoid generating REs by suppressing primary RE generation mechanisms (Dreicer [19], hot tail [20]) and reduce the avalanche [21]. However, state-of-the art models when applied to ITER 15 MA plasma conditions find finite RE primary populations are created $[15,22]$ and avalanched [21,23]. A second line of defense is therefore needed to mitigate a mature RE beam. SPI was first tested for RE mitigation on DIII-D [24] and was shown to successfully dissipate RE beam energy with high- $Z$ noble gases, building on earlier experiments with massive gas injection [25-27]. Yet, high- $Z$ mitigation was found to be ineffective in some JET experiments [10]. There is also evidence from theoretical models $[15,22,28]$ that high- $Z$ mitigation will not be sufficient for larger RE currents.

This Letter reports on the use of deuterium injections to mitigate a RE beam. For the first time, RE beams with currents up to 1.27 MA were mitigated at JET with no measurable energy deposition to the plasma facing components. This level of RE current is well in excess of levels previously found to lead to significant damage to the first wall in JET [29]. This result is achieved through a combination of the excitation of a large magnetohydrodynamic (MHD) instability followed by the absence of regeneration of REs. Qualitatively similar RE terminations were observed at DIII-D [30]. However, owing to incomplete thermography of the PFCs the DIII-D experiments were unable to assess the implications for firstwall integrity. The present work builds on those experimental findings and shows that this deconfinement method is benign for the wall despite large currents at $\mathrm{RE}$ termination. Although the avalanche amplification $\gamma_{\mathrm{RE}} t \approx$ $I_{p} /\left[I_{\text {Alfvén } \ln (\Lambda)]}[21]\right.$ is predicted to be larger for ITER than for JET, this mitigation scenario opens a new approach to dissipate REs generated during tokamak plasma disruptions.

Experimental background and runaway impact on the wall.-In this study, RE beams are created in the JET tokamak using argon injections in a limiter configuration. A standard RE scenario is shown on Fig. 1, blue curves. $2.38 \times 10^{21}$ atoms of argon are used to trigger the disruption (twice the deuterium inventory of the predisruption plasma) exciting a $750 \mathrm{kA}$ runaway beam. The companion plasma coexisting with the RE beam is mainly composed of argon from the disruption-triggering injection [31]. The impact at beam termination is characterized by infrared

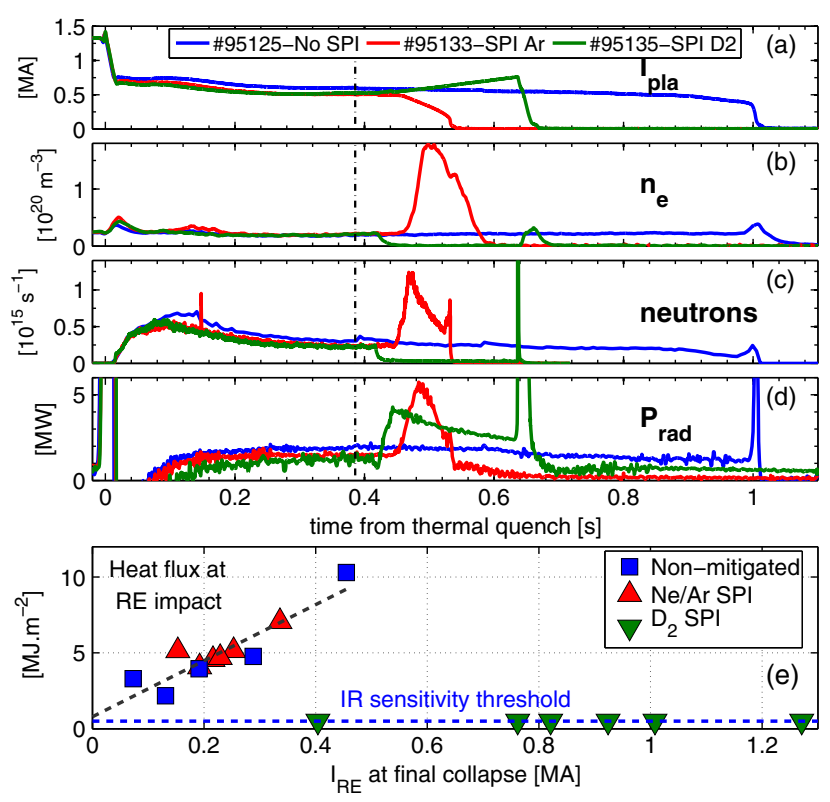

FIG. 1. Overview of three different RE beam termination scenarios. (a) Total current. (b) Average electron density. (c) Total neutron rate. (d) Total radiated power. (e) Heat flux on the wall following RE impact. Vertical dashed line is the SPI trigger time.

thermography. JET camera systems monitor the vast majority of the plasma facing components, thus ruling out the possibility of missing localized RE impacts. Heat loads are computed using a 1D finite difference heat diffusion method similar to Ref. [29], where the energy flux and the deposition depth are used to fit the measured surface temperature decay following the impact. The energy deposition duration and footprint are determined by the camera images. Injecting high- $Z$ material into the beam leads to a faster RE current decay (red curves on Fig. 1) and a final collapse producing significant heat loads on the wall [10].

Using deuterium SPI leads to completely different dynamics. On JET pulse \#95135, a shattered pellet containing $1.46 \times 10^{23}$ deuterium atoms is fired onto the $\mathrm{RE}$ beam. Instead of decreasing as with high- $Z$ injections (Fig. 1, red curves), the RE current rises [Fig. 1(a), green curve]. Free electron density drops to nonmeasurable values $\left[<10^{18} \mathrm{~m}^{-3}\right.$, Fig. 1(c)]. The neutron rate drops by a factor 10 [Fig. 1(d)]. The effect of $\mathrm{D}_{2}$ is qualitatively similar to that reported in DIII-D [24], AUG [32], and COMPASS [33], with the exception of a marked rise in the radiated power [up to $4.3 \mathrm{MW}$, Fig. 1(e)], a feature seen only on JET. The current increase is attributed to a decrease of the effective resistivity of the RE + companion plasma system. The decrease of argon line radiation and the appearance of deuterium lines are consistent with the argon being expelled from the plasma [34]. The low free electron density indicates that the plasma has recombined.

A large neutron spike occurs $220 \mathrm{~ms}$ after the shattered pellet arrival, with $\mathrm{I}_{\mathrm{RE}} \approx 760 \mathrm{kA}$. The infrared synchrotron 
emission disappears in less than $3 \mathrm{~ms}$, indicating significant loss of REs. A large magnetic perturbation is measured and a current quench similar to a conventional disruption occurs thereafter similarly to Ref. [30].

Full-view thermography of this event available at JET reveals the striking absence of any localized impact on the first wall, with heat loads below the infrared (IR) camera measurement threshold $\left(0.5 \mathrm{MJ} \mathrm{m}^{-2}\right)$. This safe termination is unique to deuterium-background RE beams as shown in Fig. 1(e) and reported here for the first time. Owing to incomplete IR camera coverage on DIII-D, only a postmortem analysis of the event reported in Ref. [30] was performed. This inspection could only conclude that no visible damage occurred to the DIII-D carbon tiles. The heat flux needed to ablate $30 \mu \mathrm{m}$ of material from a carbon tile following a $100 \mu \mathrm{s}$ pulse [30] is around $30 \mathrm{MJ} \mathrm{m}^{-2}$ from simulations [35]. It can be considered as the minimum heat loads inferrable from a postmortem analysis. This value is much larger than the $0.5 \mathrm{MJ} \mathrm{m}^{-2}$ sensitivity of the measurements reported here and too large for making reliable predictions of RE terminations in future tokamaks. The observation reported in the present Letter is therefore the first time a conclusive demonstration of the absence of heat loads can be obtained in mega-ampere scale RE beam terminations. In contrast, REs mitigated by high- $Z$ material produce significant heat loads despite lower currents at termination as shown in Fig. 1(e). The benign termination is explained by the combination of two mechanisms which will now be elaborated in turn: (1) a violent MHD instability stochastizing the plasma and (2) the absence of regeneration of REs during the subsequent current quench resulting from the expulsion of the high- $Z$ impurities.

Development of the MHD instability.-The deuterium SPI leads to an increase of the RE current and therefore a decrease of the edge safety factor $q_{\text {edge. }}$ The MHD instability triggering the final RE collapse happens when $q_{\text {edge }}$ is between 2 and 5 [high- $Z$ fraction below 0.3 on Fig. 2(a)]. The large dispersion of $q_{\text {edge }}$ at MHD onset suggests that it is not exclusively a simple current-limiting instability as proposed in Ref. [30]. Simulations of pulse \#95135 using the sOFT code [36] have been performed to reconstruct the infrared synchrotron camera images. A spatially uniform RE energy and pitch angle distribution was assumed. The best match between the simulation and the IR images is obtained when the pitch angle is between (0.1-0.3), the energy less than $15 \mathrm{MeV}$, and the RE density profile is hollow (peak density around mid-radius), as shown on Figs. 3(a) and 3(b). Using a peaked RE density profile, no energy and pitch angle distribution that explains the observed pattern on the camera image could be identified. The RE current profile therefore likely presents some degree of flatness.

Magnetic islands visible in the infrared images show that a $m=4$ surface lies at a third of the radius shortly before
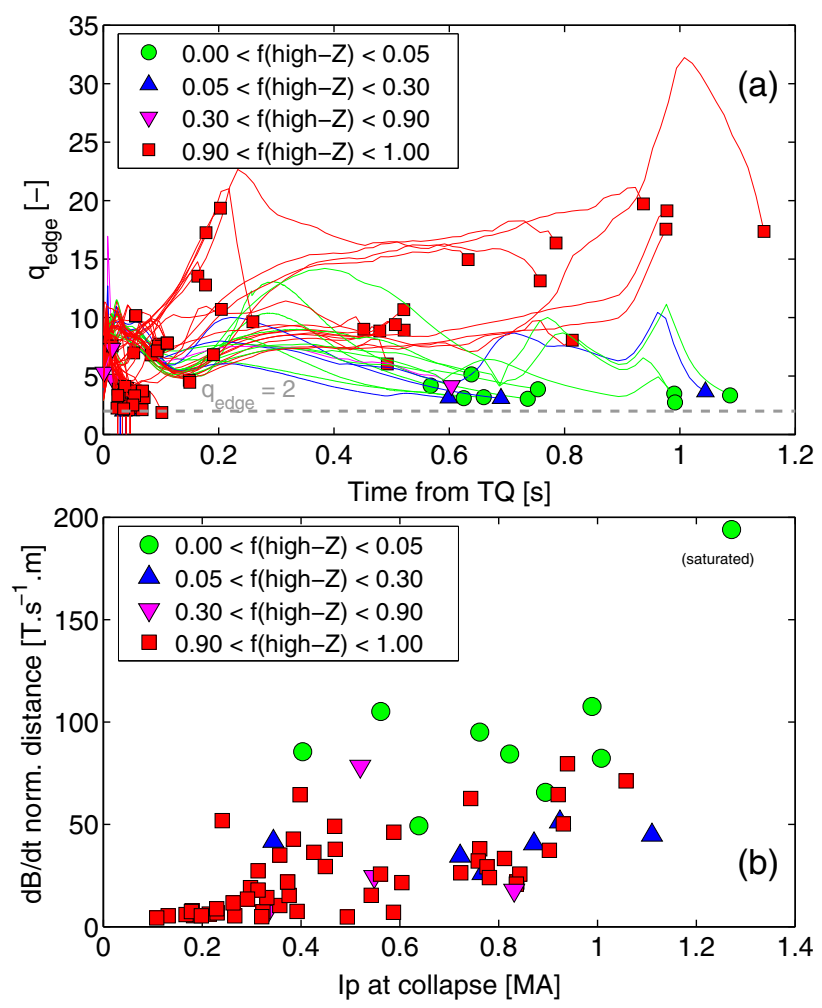

FIG. 2. Development of the collapse-inducing instability. (a) Edge safety factor of RE beams for various fractions of high- $Z$ species in mitigation material. (b) MHD perturbation amplitude normalized to the distance to the sensor versus RE current at termination.

the collapse [37]. The final instability develops without a precursor, and reaches its peak $d B / d t$ in $10-20 \mu \mathrm{s}$ as shown on Fig. 4(b). The toroidal mode number $n=1$ determined by a Mirnov coil array is the most probable, consistent with a $q=4$ instability. $q_{\text {edge }}$ is about 5.1 at this stage and the visible $q=4$ surface is close to the core $(r / a \approx 0.3)$; this is therefore further evidence for a nonmonotonic $q$ profile. Magnetohydrodyamic simulations of this RE beam using the JOREK code $[38,39]$ have been made [37] using the RE fluid model from Ref. [40] and the current profile estimated above. The dynamics are dominated by tearing mode formation at the two $q=4$ surfaces
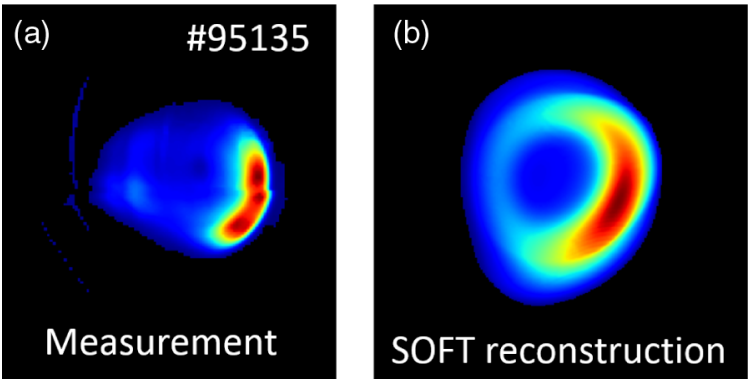

FIG. 3. (a) Measured infrared $(\lambda=3-3.5 \mu \mathrm{m})$ synchrotron radiation image. (b) Reconstructed synchrotron radiation using SOFT. 


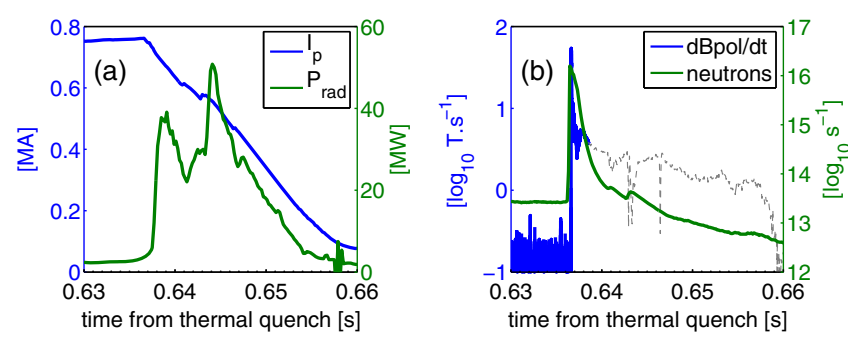

FIG. 4. Collapse of a $D_{2}$-mitigated RE beam. (a) Total current (blue) and radiated power (green). (b) Measured magnetic perturbation amplitude (blue) and neutron rate (green). The dashed part of the curve is the $n=0$ perturbation due to $\mathrm{I}_{p}$ decay.

leading to a stochastization of the magnetic field first around the outer $q=4$ surface, followed by the shrinking of the core and the destruction of the entire confinement in about $100 \mu$ s (Fig. 5) on a timescale consistent with the experimental measurements of magnetic fluctuations. During the burst of MHD activity, more than $95 \%$ of the REs are lost in the simulation and the current is converted into thermal current while a small current spike appears. The current profile is strongly flattened by fast magnetic reconnection during the MHD event. The simulated RE loss area near the contact point is widened by the edge stochastization.

The normalized growth rate $d B_{\text {pol }} / d t$ of the instability measured experimentally is compared between cases where a significant energy is deposited by REs (natural and high$Z$ injections) and cases with no measurable impacts on Fig. 2(b). The growth rate of the perturbation is larger for $\mathrm{D}_{2}$-mitigated than high- $Z$-mitigated beams, but with overlap. The magnitude $\delta B_{\text {pol }} / B_{\text {pol }}$ of the instability is even less correlated with wall heat loads. The short timescale of the instability in $\mathrm{D}_{2}$ cases is therefore a key ingredient of the large RE loss, but not sufficient to explain the absence of heat loads.

Regeneration of runaway electrons.-The prompt loss of REs is followed by a current quench. The current carriers

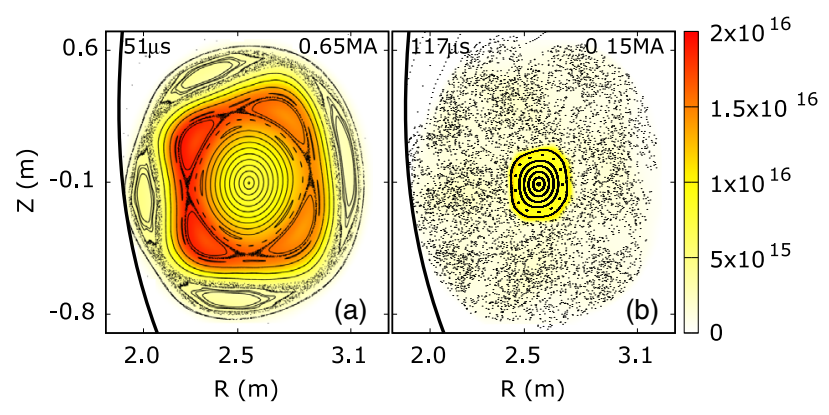

FIG. 5. Poincare plots at different times in the JOREK simulation showing double tearing mode formation and stochastization starting from the edge. REs are largely lost during the MHD event. (a) $t=51 \mu \mathrm{s}$, (b) $t=117 \mu \mathrm{s}$. Early nonlinear phase is taken as reference time $t=0$. Background color scale represents RE density $n_{r}$. shift from REs to bulk plasma, thus leading to plasma reionization and a $50 \mathrm{MW}$ radiated power spike [Fig. 4(a)]. Line radiation of weakly ionized argon dominates the current quench, indicating that the argon is not completely purged. For higher argon concentrations some REs are regenerated: a few milliseconds after the initial termination, a small RE beam reappears as evidenced by synchrotron emission (Fig. 6). The current quench rate and the maximum radiated power normalized to the initial magnetic energy are correlated with the ratio of the amount of argon (used to trigger the disruption) to deuterium (in the SPI) as shown in Fig. 7(a). This behavior can be investigated through the following model. In the absence of impurities, the Ohmic reheating of cold plasma is faster than Dreicer and avalanche mechanisms of $\mathrm{RE}$ production [41]. If enough impurities remain in the plasma, reheating is hindered by line radiation and the persistent electric field facilitates the RE avalanche. The system of equations governing energy balance, vessel current $I_{v}$, total plasma current $I$, and RE current $I_{\mathrm{RE}}$ is solved numerically to analyze the plasma temperature and current evolution selfconsistently with the RE generation $[42,43]$ :

$$
\begin{aligned}
\frac{3}{2} \frac{\partial}{\partial t} n_{f} T_{e} & =\frac{\left(I-I_{\mathrm{RE}}\right)^{2}}{\sigma S^{2}}-n_{f} n_{Z} L\left(T_{e}\right) \\
\frac{d}{d t}\left(L I+L_{v} I_{v}\right) & =-2 \pi R E \\
\frac{d}{d t}\left(L_{v} I+L_{v} I_{v}\right) & =-I_{v} R_{v} \\
\frac{1}{I_{\mathrm{RE}}} \frac{\partial I_{\mathrm{RE}}}{\partial t} \approx & \frac{n_{f}+n_{b}}{n_{f} \ln \Lambda_{f}\left(p_{c}\right)+n_{b} \ln \Lambda_{b}\left(p_{c}\right)} \cdots \\
& \cdots \frac{1}{\sqrt{Z_{\mathrm{RE}}\left(p_{c}\right)+5}} \frac{e\left(E-E_{\mathrm{crit}}\right)}{m_{e} c}
\end{aligned}
$$

where the free electron density $n_{f}=n_{D}+n_{Z} Z\left(T_{e}\right), n_{D}$ and $n_{Z}$ are deuterium and impurity densities, $Z\left(T_{e}\right)$ and $L\left(T_{e}\right)$ are the mean impurity ionization level and radiation efficiency [44], $\sigma$ the Spitzer conductivity, $S$ the poloidal cross section area, and $R$ the major radius. The mutual plasma-vessel inductance is taken equal to the vessel

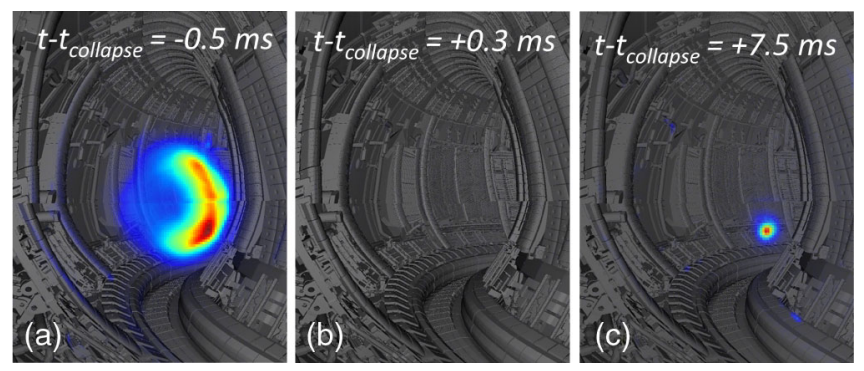

FIG. 6. IR synchrotron emission images (a), just before the RE dissipation (b) just after the RE dissipation, (c) during the final collapse, showing regenerated REs. 

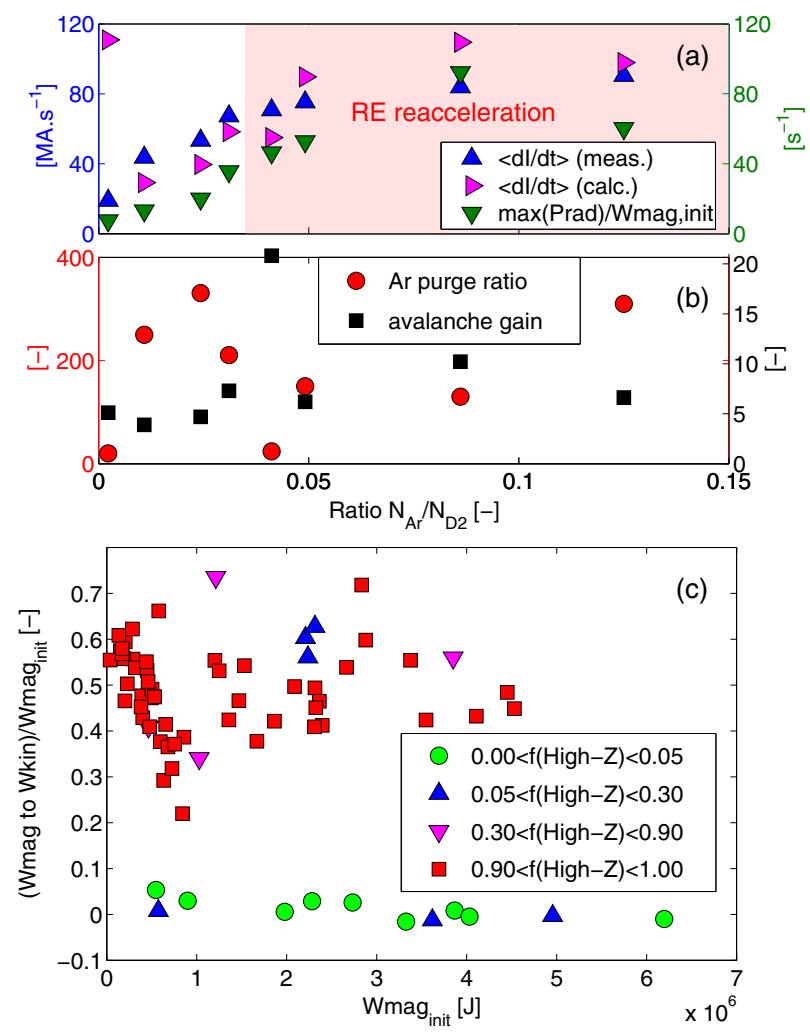

FIG. 7. Effect of the high- $Z$ impurity content on the RE collapse. (a) Current decay rate (measured and calculated by the model), maximum radiated power of the final collapse versus $\mathrm{Ar} / \mathrm{D}_{2}$ ratio from injection material. (b) Calculated argon purge ratio needed to match the radiated power and avalanche gain. (c) Fraction of RE magnetic energy converted into RE kinetic energy during the final collapse versus precollapse magnetic energy for various fractions of high- $Z$ impurities from injection material.

inductance $L_{v}$, while the plasma inductance $L \approx L_{i}+L_{v}$ (with $L_{i} \approx 0.5 \mu_{0} R l_{i}$ being the internal plasma inductance). The Rosenbluth-Putvinski avalanche formula [21] is modified to include the effect of partially ionized impurities $[28,45]$, i.e., only a fraction of bound electrons contributes to the friction of fast particles. This fraction is given by the ratio of Coulomb logarithms for free $\left[\ln \Lambda_{f}\left(p_{c}\right)\right]$ and bound $\left[\ln \Lambda_{b}\left(p_{c}\right)\right]$ electron collisions. In the simulations the quantities $\ln \Lambda_{b}\left(p_{c}\right), \ln \Lambda_{f}\left(p_{c}\right)$, and $Z_{\mathrm{RE}}\left(p_{c}\right)$ are taken from Eqs. (27), (29), and (40) of Ref. [41], respectively. The critical momentum $p_{c}(E)$ is obtained from the acceleration-friction force balance equation. Deuterium density is estimated from the injection amount, while the residual argon density $n_{Z}$ is chosen such that the maximum radiated power matches the experiment. With the vacuum vessel resistive time $\tau_{v}=L_{v} / R_{v} \approx 5 \mathrm{~ms}$ [46] and its inductance $L_{v} \approx 2 \mu \mathrm{H}$, the trend in current quench rates obtained from the calculations agrees qualitatively with the experimental measurements [Fig. 7(a)]. Figure 7(b) shows that the argon purge rate $N_{\text {Ar,initial }} / N_{\text {Ar,final }}$ is between 50 and 300 . The calculated avalanche gain is too low to regenerate a full RE beam in these cases but the small increasing trend with $\mathrm{Ar} / \mathrm{D}_{2}$ ratio in Fig. 7(b) highlights the role of residual impurities in the RE regeneration. The cases of pure high- $Z$ SPI can thus be seen as limit cases in which regeneration occurs continuously during the collapse. Note that an ITER full current case would require a larger purge ratio due to the higher RE avalanche amplification.

Continuous RE regeneration during collapse plays a decisive role in the process of conversion of magnetic energy (current carried by REs) into kinetic energy deposited on the wall. For the case presented in Figs. 4(a) and 4(b), the magnetic energy $W_{\text {mag }} \approx 2.2 \mathrm{MJ}$ is larger than the kinetic energy $W_{\text {kin }} \approx 0.4 \mathrm{MJ}$. Therefore damage may arise if a large fraction of $W_{\text {mag }}$ is converted into $W_{\text {kin }}[15,16]$. The conversion rate calculated using the method proposed in Ref. [16] adding radiated power as a loss term is shown in Fig. 7(c). The conversion rate is close to zero for cases where the companion plasma contains less than $30 \%$ of high- $Z$ impurities. Low $-Z$ and high- $Z$ cases are clearly distinguished showing that benign terminations due to deuterium SPI rely on the weak conversion of $W_{\text {mag }}$ to $W_{\text {kin }}$.

Discussion.-A scenario leading to a safe termination of large RE beams is found to be efficient and reproducible on JET. Further experiments are planned to explore the minimum level of companion plasma purity needed to achieve the safe termination scenario and to investigate the conditions in which the MHD instability develops. The applicability of such scenarios to larger tokamaks is an open question: larger avalanche gains are predicted for ITER, therefore simulations are required to assess the accessibility of a regime with a large enough MHD instability and purge ratio. The role of magnetic reconnection will also be investigated further: MHD reconnection is known to lead to such energy conversion in solar plasmas [47], but its role in tokamak plasmas is more ambiguous: it can lead to conversion [48] or the absence of it as reported in the present Letter. Nonetheless, even if some runaway regeneration occurs during the final MHD collapse, only a fraction of the precollapse current will be converted back to REs via the avalanche. It is therefore conceivable that a sequence of harmless RE beam quenches could ensure a safe termination of an arbitrarily large RE beam by multiple deuterium SPIs leading to a stepwise reduction of RE current below the damage threshold. The deuterium RE mitigation scenario reported in the present Letter could therefore potentially solve one of the major issues of future reactors based on the tokamak concept.

This work has been carried out within the framework of the EUROfusion Consortium and has received funding from the Euratom research and training programme 20142018 and 2019-2020 under Grant Agreement No. 633053. The views and opinions expressed herein do not necessarily 
reflect those of the European Commission. Some of the simulations presented in this article were performed using the Marconi-Fusion supercomputer. This work was supported by the EUROfusion-Theory and Advanced Simulation Coordination (E-TASC). This material is based upon work supported by the U.S. Department of Energy, Office of Science, Office of Fusion Energy Sciences, under Award No. DE-SC0020299.

*Corresponding author.

cedric.reux@cea.fr

'See the author list of "Overview of JET results for optimising ITER operation" by J. Mailloux et al. to be published in Nucl. Fus. Special issue: Overview and Summary Papers from the 28th Fusion Energy Conference (Nice, France, 10-15 May 2021).

[1] C. T. R. Wilson, The acceleration of $\beta$-particles in strong electric fields such as those of thunderclouds, Math. Proc. Cambridge Philos. Soc. 22, 534 (1925).

[2] A. Gurevich, G. Milikh, and R. Roussel-Dupre, Runaway electron mechanism of air breakdown and preconditioning during a thunderstorm, Phys. Lett. A 165, 463 (1992).

[3] G. Haerendel, Evidence for field-parallel electron acceleration in solar flares, Astrophys. J. 847, 113 (2017).

[4] G. D. Holman, Acceleration of runaway electrons and joule heating in solar flares, in Unstable Current Systems and Plasma Instabilities in Astrophysics, edited by M. R. Kundu and G. D. Holman (Springer, Dordrecht, Netherlands, 1985), pp. 191-196.

[5] E. P. Gorbunov and K. A. Razumova, Effect of a strong magnetic field on the magnetohydrodynamic stability of a plasma and the confinement of charged particles in the tokamak machine, J. Nucl. Energy, Part C 6, 515 (1964).

[6] D. Spicer, An unstable arch model of a solar flare, Sol. Phys. 53, 305 (1977).

[7] J. Guo, A. G. Emslie, and M. Piana, The specific acceleration rate in loop-structured solar flares-Implications for electron acceleration models, Astrophys. J. 766, 28 (2013).

[8] L.-G. Eriksson, P. Helander, F. Andersson, D. Anderson, and M. Lisak, Current Dynamics During Disruptions in Large Tokamaks, Phys. Rev. Lett. 92, 205004 (2004).

[9] R. Gill, B. Alper, A. Edwards, L. Ingesson, M. Johnson, and D. Ward, Direct observations of runaway electrons during disruptions in the JET tokamak, Nucl. Fusion 40, 163 (2000).

[10] C. Reux et al., Runaway electron beam generation and mitigation during disruptions at JET-ILW, Nucl. Fusion 55, 093013 (2015).

[11] E. Hollmann et al., Study of argon assimilation into the post-disruption runaway electron plateau in DIII-D and comparison with a 1d diffusion model, Nucl. Fusion 59, 106014 (2019).

[12] F. Saint-Laurent, G. Martin, T. Alarcon, A. Le Luyer, P. Parks, P. Pastor, S. Putvinski, C. Reux, J. Bucalossi, S. Bremond, and P. Moreau, Overview of runaway electron control and mitigation experiments on Tore Supra and lessons learned in view of iter, Fusion Sci. Technol. 64, 711 (2013).
[13] R. Nygren, T. Lutz, D. Walsh, G. Martin, M. Chatelier, T. Loarer, and D. Guilhem, Runaway electron damage to the Tore Supra phase III outboard pump limiter, J. Nucl. Mater. 241-243, 522 (1997).

[14] G. F. Matthews, B. Bazylev, A. Baron-Wiechec, J. Coenen, K. Heinola, V. Kiptily, H. Maier, C. Reux, V. Riccardo, F. Rimini, G. Sergienko, V. Thompson, and A. W. and, Melt damage to the JET ITER-like wall and divertor, Phys. Scr. T167, 014070 (2016).

[15] J. Martín-Solís, A. Loarte, and M. Lehnen, Formation and termination of runaway beams in ITER disruptions, Nucl. Fusion 57, 066025 (2017).

[16] A. Loarte, V. Riccardo, J. Martin-Solís, J. Paley, A. Huber, and M. L. and, Magnetic energy flows during the current quench and termination of disruptions with runaway current plateau formation in JET and implications for ITER, Nucl. Fusion 51, 073004 (2011).

[17] M. Lehnen et al., Disruptions in ITER and strategies for their control and mitigation, J. Nucl. Mater. 463, 39 (2015).

[18] L. Baylor, S. Meitner, T. Gebhart, J. Caughman, J. Herfindal, D. Shiraki, and D. Youchison, Shattered pellet injection technology design and characterization for disruption mitigation experiments, Nucl. Fusion 59, 066008 (2019).

[19] H. Dreicer, Electron and ion runaway in a fully ionized gas. I, Phys. Rev. 115, 238 (1959).

[20] H. M. Smith and E. Verwichte, Hot tail runaway electron generation in tokamak disruptions, Phys. Plasmas 15, 072502 (2008).

[21] M. Rosenbluth and S. Putvinski, Theory for avalanche of runaway electrons in tokamaks, Nucl. Fusion 37, 1355 (1997).

[22] O. Vallhagen, O. Embreus, I. Pusztai, L. Hesslow, and T. Fülöp, Runaway dynamics in ITER-like disruptions in the presence of massive material injection, J. Plasma Phys. 86, 475860401 (2020).

[23] T. Fülöp, O. Embréus, A. Stahl, S. Newton, I. Pusztai, and G. Wilkie, Kinetic modelling of runaways in fusion plasmas, in 26th IAEA Fusion Energy Conference (International Atomic Energy Agency (IAEA), 2016), p. 066008.

[24] D. Shiraki, N. Commaux, L. Baylor, C. Cooper, N. Eidietis, E. Hollmann, C. Paz-Soldan, S. Combs, and S. Meitner, Dissipation of post-disruption runaway electron plateaus by shattered pellet injection in DIII-D, Nucl. Fusion 58, 056006 (2018).

[25] D. G. Whyte, T. C. Jernigan, D. A. Humphreys, A. W. Hyatt, C. J. Lasnier, P. B. Parks, T. E. Evans, M. N. Rosenbluth, P. L. Taylor, A. G. Kellman, D. S. Gray, E. M. Hollmann, and S. K. Combs, Mitigation of Tokamak Disruptions Using High-Pressure Gas Injection, Phys. Rev. Lett. 89, 055001 (2002).

[26] E. Hollmann et al., Control and dissipation of runaway electron beams created during rapid shutdown experiments in DIII-D, Nucl. Fusion 53, 083004 (2013).

[27] F. Saint-Laurent, C. Reux, J. Bucalossi, A. Loarte, S. Brémond, C. Gil, P. Moreau, and J.-L. Segui, Control of runaway electron beams on Tore Supra, 36th EPS Conference on Plasma Phys. Sofia (European Physical Society, 2009), p. 4.205.

[28] L. Hesslow, O. Embréus, O. Vallhagen, and T. Fülöp, Influence of massive material injection on avalanche 
runaway generation during tokamak disruptions, Nucl. Fusion 59, 084004 (2019).

[29] C. Reux et al., Runaway beam studies during disruptions at JET-ILW, J. Nucl. Mater. 463, 143 (2015).

[30] C. Paz-Soldan, N. W. Eidietis, Y. Q. Liu, D. Shiraki, A. H. Boozer, E. M. Hollmann, C. C. Kim, and A. Lvovskiy, Kink instabilities of the post-disruption runaway electron beam at low safety factor, Plasma Phys. Controlled Fusion 61, 054001 (2019).

[31] S. Sridhar, C. Reux, P. Beyer, M. Lehnen, I. Coffey, R. Guirlet, and N. Fedorczak (JET contributors), Characterization of cold background plasma during the runaway electron beam mitigation experiments in the JET tokamak, Nucl. Fusion 60, 096010 (2020).

[32] G. Pautasso, M. Dibon, M. Dunne, R. Dux, E. Fable, P. Lang, O. Linder, A. Mlynek, G. Papp, M. Bernert, A. Gude, M. Lehnen, P. McCarthy, and J. Stober, Generation and dissipation of runaway electrons in ASDEX upgrade experiments, Nucl. Fusion 60, 086011 (2020).

[33] J. Mlynar et al., Runaway electron experiments at COMPASS in support of the EUROfusion ITER physics research, Plasma Phys. Controlled Fusion 61, 014010 (2019).

[34] E. M. Hollmann, I. Bykov, N. W. Eidietis, J. L. Herfindal, A. Lvovskiy, R. A. Moyer, P. B. Parks, C. Paz-Soldan, A. Y. Pigarov, D. L. Rudakov, D. Shiraki, and J. Watkins, Study of argon expulsion from the post-disruption runaway electron plateau following low-z massive gas injection in DIII-D, Phys. Plasmas 27, 042515 (2020).

[35] H. Pacher, I. Smid, G. Federici, Y. Igitkhanov, G. Janeschitz, R. Raffray, and G. Vieider, Erosion lifetime of ITER divertor plates, J. Nucl. Mater. 241-243, 255 (1997).

[36] M. Hoppe, O. Embréus, R. Tinguely, R. Granetz, A. Stahl, and T. Fülöp, SOFT: A synthetic synchrotron diagnostic for runaway electrons, Nucl. Fusion 58, 026032 (2018).

[37] V. Bandaru, M. Hoelzl, C. Reux, O. Ficker, S. Silburn, M. Lehnen, N. Eidietis, and J. Team, Magnetohydrodynamic simulations of runaway electron beam termination in JET, Plasma Phys. Controlled Fusion 63, 035024 (2021).

[38] G. Huysmans and O. Czarny, MHD stability in x-point geometry: Simulation of ELMs, Nucl. Fusion 47, 659 (2007).
[39] M. Hoelzl et al., The JOREK non-linear extended MHD code and applications to large-scale instabilities and their control in magnetically confined fusion plasmas, arXiv: 2011.09120 [Nucl. Fusion (to be published)].

[40] V. Bandaru, M. Hoelzl, F. J. Artola, G. Papp, and G. T. A. Huijsmans, Simulating the nonlinear interaction of relativistic electrons and tokamak plasma instabilities: Implementation and validation of a fluid model, Phys. Rev. E 99, 063317 (2019).

[41] B. N. Breizman, P. Aleynikov, E. M. Hollmann, and M. Lehnen, Physics of runaway electrons in tokamaks, Nucl. Fusion 59, 083001 (2019).

[42] J. R. Martin-Solis, A. Loarte, and M. Lehnen, On the avalanche generation of runaway electrons during tokamak disruptions, Phys. Plasmas 22, 082503 (2015).

[43] P. Aleynikov and B. N. Breizman, Generation of runaway electrons during the thermal quench in tokamaks, Nucl. Fusion 57, 046009 (2017).

[44] H. Summers, M. O’Mullane, A. Whiteford, N. Badnell, and S. Loch, ADAS: Atomic data, modelling and analysis for fusion, Ame. Inst. Phys. 901, 239 (2007).

[45] J. R. Martín-Solís, A. Loarte, and M. Lehnen, Runaway electron dynamics in tokamak plasmas with high impurity content, Phys. Plasmas 22, 092512 (2015).

[46] R. Albanese, M. Mattei, and F. Villone, Prediction of the growth rates of VDEs in JET, Nucl. Fusion 44, 999 (2004).

[47] V. V. Zharkova, A. O. Arzner, K. Benz, P. Browning, C. Dauphin, A. G. Emslie, L. Fletcher, E. P. Kontar, G. Mann, M. Onofri, V. Petrosian, R. Turkmani, N. Vilmer, and L. Vlahos, Recent advances in understanding particle acceleration processes in solar flares, Space Sci. Rev. 159, 357 (2011).

[48] S. J. Freethy, K. G. McClements, S. C. Chapman, R. O. Dendy, W. N. Lai, S. J. P. Pamela, V. F. Shevchenko, and R. G. L. Vann, Electron Kinetics Inferred from Observations of Microwave Bursts During Edge Localized Modes in the Mega-amp Spherical Tokamak, Phys. Rev. Lett. 114, 125004 (2015). 